Copyright (C) 2013 IEEE. Personal use of this material is permitted. Permission from IEEE must be obtained for all other uses, in any current or future media, including reprinting/republishing this material for advertising or promotional purposes, creating new collective works, for resale or redistribution to servers or lists, or reuse of any copyrighted component of this work in other works. 


\title{
On the Design of Amplify-and-Forward MIMO-OFDM Relay Systems with QoS Requirements Specif ed as Schur-convex Functions of the MSEs
}

\author{
Luca Sanguinetti, Member, IEEE, Antonio A. D'Amico and Yue Rong, Senior Member, IEEE.
}

\begin{abstract}
In this letter, we focus on the design of linear and non-linear architectures in amplify-and-forward multiple-input multiple-output orthogonal frequency-division multiplexing relay networks in which different types of services are supported. The goal is to jointly optimize the processing matrices so as to minimize the total power consumption while satisfying the quality-of-service requirements of each service specified as Schurconvex functions of the mean square errors over all assigned subcarriers. It turns out that the optimal solution leads to the diagonalization of the source-relay-destination channel up to a unitary matrix depending on the specific Schur-convex function.
\end{abstract}

Index Terms-MIMO, OFDM, non-regenerative relay, qualityof-service requirements, transceiver design, Schur-convex functions, power minimization, amplify-and-forward.

\section{INTRODUCTION}

Over the last years, the ever-increasing demand for highspeed ubiquitous wireless communications has motivated an intense research activity towards the development of transmission technologies characterized by high spectral eff ciency and high reliability. The most promising solutions in this direction rely on orthogonal-frequency division-multiplexing (OFDM) techniques, multiple-input multiple-output (MIMO) schemes, and relay-assisted communications [1] - [2]. This is witnessed by the adoption of all these technologies in recent standards such as 3GPPs LTE [3] and IEEE 802.16j [4].

In this context, the optimization of linear as well as non-linear architectures for MIMO or MIMO-OFDM nonregenerative relay networks has received much attention recently (see for example [5] - [16] and references therein). Most of the existing works can be largely categorized into two different classes. The $\mathrm{f}$ rst one is focused on the minimization/maximization of a global objective function subject to average power constraints at the source and relay nodes (see for example [8] - [9]) while the second aims at minimizing the total power consumption under specif c quality-of-service (QoS) requirements (see for example [11] and references therein). In particular, in [11] the authors make use of majorization theory and propose a unifying framework for minimizing the total

L. Sanguinetti and Antonio A. D'Amico are with the Department of Information Engineering, University of Pisa, Via Caruso 56126 Pisa, Italy (e-mail: \{luca.sanguinetti, a.damico\}@iet.unipi.it).

Yue Rong is with the Department of Electrical and Computer Engineering, Curtin University of Technology, Bentley, WA 6102, Australia (e-mail: y.rong@curtin.edu.au). power consumption in linear and non-linear multi-hop MIMO relay systems while meeting specif c QoS requirements given in terms of the mean-square-errors (MSEs) over the different streams. Denoting by $K$ the number of streams, the above optimization problem can be mathematically formulated as [11]

$$
\min P_{T} \quad \text { s.t. } \quad \mathrm{MSE}_{k} \leq \gamma_{k} \quad \forall k \in \mathcal{K}
$$

where $\mathcal{K}=\{1,2, \ldots, K\}, P_{T}$ denotes the total power consumption, $\mathrm{MSE}_{k}$ is the MSE of the $k$ th stream and the quantities $\left\{\gamma_{k}\right\}$ are design parameters that specify the different stream requirements. The minimization is performed with respect to the processing matrices at the source, relay and destination nodes. Similar to [5] - [9], in [11] it is shown that the solution of (1) leads to the diagonalization of the sourcerelay-destination channel. The extension of the above problem to MIMO-OFDM relay systems is discussed in [14] (see also [11] and [15]) in which the following problem is considered

$$
\min P_{T} \text { s.t. } \operatorname{MSE}_{k}(n) \leq \gamma_{k}(n) \quad \forall k \in \mathcal{K} \quad \forall n \in \mathcal{N}
$$

where $\mathcal{N}=\{1,2, \ldots, N\}$ with $N$ being the number of subcarriers whereas $\operatorname{MSE}_{k}(n)$ denotes the MSE of the $k$ th stream over the $n$th subcarrier and $\gamma_{k}(n)$ its corresponding QoS requirement. As discussed in [14], the solution of (2) can be computed following the same steps illustrated in [11] since the formulation in (2) is substantially equivalent to the one given in (1) with the only difference that each stream is required to satisfy individual QoS constraints over each subcarrier.

\section{A. Motivation}

Although reasonable, the formulation in (2) may prevent its applicability to practical OFDM applications. To see how this comes about, observe that in OFDM systems the information bits associated to each service are frst fed to an encoder (in order to exploit the frequency selectivity of the channel) and then mapped onto complex-valued symbols taken from $L$-ary constellations. The obtained symbols are eventually passed to an OFDM modulator and launched over the multipath channel. At the destination, the received signal is fed to an OFDM demodulator where the different streams are frst separated and then passed to a decoder. From the above discussion, it easily follows that the reliability of each service depends on a global performance metric measured over the assigned subcarriers rather than on individual constraints over each subcarrier. Since many different optimization criteria driving the design 
of wireless communication systems arise in connection with Schur-convex functions (see [17] for a detailed discussion on the subject), in this work we aim at solving the following problem

$$
\min \quad P_{T} \quad \text { s.t. } \quad f_{k}\left(\operatorname{MSE}_{k}(n) ; \forall n \in \mathcal{N}\right) \leq \gamma_{k} \quad \forall k \in \mathcal{K}
$$

where $f_{k}$ is a generic additively or multiplicatively Schurconvex function [18]. The only difference between (2) and (3) is represented by the QoS constraints that are in (3) specif ed as Schur-convex functions of the MSEs for the $k$ th stream over all used subcarriers. This makes (3) not only mathematically different from (2) but also more interesting from a practical point of view. Our formulation allows to embrace most of the QoS requirements that can be imposed in the design of MIMO-OFDM systems. As shown later (see also [17] for more details), they can be interpreted as the reliability constraints that in multimedia MIMO-OFDM applications are imposed on a global performance metric of the MSEs, signal-to-noise ratios (SINRs) or bit-error-rates (BERs) over all the subcarriers assigned to each service. This is surely more practical and meaningful than requiring to fulf 11 individual QoS constraints over each subcarrier as it is required in (2).

\section{B. Contribution}

To the best of our knowledge, this is the frst time that the optimization of MIMO-OFDM relay systems with QoS constraints given as Schur-convex functions of the MSEs is studied. In addition, the solution of (3) cannot be obtained using the mathematical arguments illustrated in [11] and we are not aware of any existing work in which the solution of (3) is provided. The major contribution of this work is to rigorously prove that the solution of (3) leads to the diagonalization of the source-relay-destination channel up to a unitary matrix. Differently from [11] and [15], the latter is found to be such that the individual MSEs are all equal to a quantity depending on the specif c Schur-convex function ${ }^{1}$. Once the solution of (3) is proven to be such that the source-relay-destination channel is diagonalized up to an unitary matrix, the power minimization problem in (3) reduces to properly allocating the available power over the established links. Solving such a problem is out of the scope of the submitted letter since its solution can be found with affordable complexity resorting for example to the power allocation algorithm developed in [15]. For simplicity, we focus only on a two-hop system in which a single relay is employed. However, all the provided results can be easily extended to a multi-hop scenario and clearly to conventional single-hop MIMO-OFDM systems [19].

\section{SYSTEM DESCRIPTION}

We consider a MIMO-OFDM relay network in which $N$ subcarriers out of the total number $N_{T}$ are used to support

\footnotetext{
${ }^{1}$ It is important to remark that the results of this work are valid only for Schur-convex functions. For example, they do not hold true for Schurconcave functions (see [17] for more details). Although f nite, the set of Schur-convex functions is still of much importance as it embraces most of the QoS requirements that can be imposed in the design of MIMO-OFDM applications.
}

$K$ different classes of services ${ }^{2}$. The source and destination are equipped with $N_{S}$ antennas while the relay has $N_{R}$ antennas. The $k$ th symbol over the $n$th subcarrier is denoted by $s_{k}(n)$ and is taken from an $L$-ary quadrature amplitude modulation constellation with average power normalized to unity for convenience.

The input data stream is divided into adjacent blocks of $N K \leq \min \left(N N_{R}, N N_{S}\right)$ symbols, which are transmitted in parallel using the $N$ assigned subcarriers with indices $\left\{i_{n} ; n=1,2, \ldots, N\right\}$. The vector $\mathbf{s}=\left[\mathbf{s}_{1}^{T}, \mathbf{s}_{2}^{T}, \ldots, \mathbf{s}_{K}^{T}\right]^{T}$ with $\mathbf{s}_{k}=\left[s_{k}(1), s_{k}(2), \ldots, s_{k}(N)\right]^{T}$ is f rst linearly processed by a matrix $\mathbf{U} \in \mathbb{C}^{N N_{S} \times K N}$ and then launched over the the source-relay MIMO channel using $N_{S}$ OFDM modulators. At the relay, the received signal is processed by a matrix $\mathbf{F} \in \mathbb{C}^{N N_{R} \times N N_{R}}$ and forwarded to the destination where the vector $\mathbf{r} \in \mathbb{C}^{N N_{S} \times 1}$ at the output of the $N_{S}$ OFDM demodulators takes the form

$$
\mathbf{r}=\mathbf{H U} \mathbf{s}+\mathbf{n}
$$

where $\mathbf{H}=\mathbf{H}_{2} \mathbf{F} \mathbf{H}_{1}$ is the equivalent channel matrix. In addition, $\mathbf{H}_{1} \in \mathbb{C}^{N N_{R} \times N N_{S}}$ and $\mathbf{H}_{2} \in \mathbb{C}^{N N_{S} \times N N_{R}}$ denote the source-relay and relay-destination block diagonal channel matrices given by

$$
\mathbf{H}_{1}=\operatorname{blkdiag}\left\{\mathbf{H}_{1}\left(i_{1}\right), \mathbf{H}_{1}\left(i_{2}\right), \ldots, \mathbf{H}_{1}\left(i_{N}\right)\right\}
$$

and

$$
\mathbf{H}_{2}=\operatorname{blkdiag}\left\{\mathbf{H}_{2}\left(i_{1}\right), \mathbf{H}_{2}\left(i_{2}\right), \ldots, \mathbf{H}_{2}\left(i_{N}\right)\right\}
$$

with $\mathbf{H}_{1}\left(i_{n}\right) \in \mathbb{C}^{N_{R} \times N_{S}}$ and $\mathbf{H}_{2}\left(i_{n}\right) \in \mathbb{C}^{N_{S} \times N_{R}}$ being the channel matrices over the $n$th subcarrier of the corresponding link. In addition, $\mathbf{n} \in \mathbb{C}^{N N_{S} \times 1}$ is a Gaussian vector with zero mean and covariance matrix $\mathbf{R}_{\mathbf{n}}=\rho_{1} \mathbf{H}_{2} \mathbf{F} \mathbf{F}^{H} \mathbf{H}_{2}^{H}+\rho_{2} \mathbf{I}_{N N_{S}}$ with $\rho_{1}>0$ and $\rho_{2}>0$ being the noise variance over each link. Henceforth, we denote by

$$
\mathbf{H}_{1}=\boldsymbol{\Omega}_{H_{1}} \boldsymbol{\Lambda}_{H_{1}}^{1 / 2} \mathbf{V}_{H_{1}}^{H} \quad \text { and } \quad \mathbf{H}_{2}=\boldsymbol{\Omega}_{H_{2}} \boldsymbol{\Lambda}_{H_{2}}^{1 / 2} \mathbf{V}_{H_{2}}^{H}
$$

the singular value decompositions of $\mathbf{H}_{1}$ and $\mathbf{H}_{2}$ and assume that the entries of the diagonal matrices $\boldsymbol{\Lambda}_{H_{1}}$ and $\boldsymbol{\Lambda}_{H_{2}}$ are in decreasing order.

\section{OPTIMIZATION OF THE RELAY NETWORK}

As mentioned previously, the goal of this work is to fnd the processing matrices that solve (3) where $P_{T}$ takes the form [11]

$$
P_{T}=\operatorname{tr}\left\{\mathbf{U} \mathbf{U}^{H}+\mathbf{F}\left(\mathbf{H}_{1} \mathbf{U} \mathbf{U}^{H} \mathbf{H}_{1}{ }^{H}+\rho_{1} \mathbf{I}_{N N_{R}}\right) \mathbf{F}^{H}\right\}
$$

while $f_{k}$ is either an additively or a multiplicatively Schurconvex function.

\footnotetext{
${ }^{2}$ The following notation is used throughout the letter. Boldface upper and lower-case letters denote matrices and vectors, respectively, while lowercase letters denote scalars. We use $\mathbf{A}=\operatorname{diag}\left\{a_{1}, a_{2}, \ldots, a_{K}\right\}$ to indicate a $K \times K$ diagonal matrix with entries $a_{k}$ for $k=1,2, \ldots, K$ and $\mathbf{A}=\operatorname{blkdiag}\left\{\mathbf{A}_{1}, \mathbf{A}_{2}, \ldots, \mathbf{A}_{K}\right\}$ to denote a block diagonal matrix. The notations $\mathbf{A}^{-1}$ and $\mathbf{A}^{1 / 2}$ denote the inverse and square-root of a matrix A. We use $\mathbf{I}_{K}$ to denote the identity matrix of order $K$ while $[\cdot]_{k, \ell}$ indicates the $(k, \ell)$ th entry of the enclosed matrix. In addition, we use $\mathrm{E}\{\cdot\}$ for expectation, the superscript ${ }^{T}$ and ${ }^{H}$ respectively for transposition and Hermitian transposition.
} 
TABLE I

LIST OF SCHUR CONVEX FUNCTIONS

\begin{tabular}{|c||c|}
\hline The sum of the MSEs & $f_{k}\left(\left\{\left[\mathbf{E}_{k}\right]_{n, n}\right\}_{n=1}^{N}\right)=\sum_{n=1}^{N}\left[\mathbf{E}_{k}\right]_{n, n}$ \\
\hline The geometric mean of the MSEs & $f_{k}\left(\left\{\left[\mathbf{E}_{k}\right]_{n, n}\right\}_{n=1}^{N}\right)=\prod_{n=1}^{N}\left[\mathbf{E}_{k}\right]_{n, n}$ \\
\hline The maximum of the MSEs & $f_{k}\left(\left\{\left[\mathbf{E}_{k}\right]_{n, n}\right\}_{n=1}^{N}\right)=\max _{1 \leq n \leq N}\left[\mathbf{E}_{k}\right]_{n, n}$ \\
\hline The harmonic mean of the SINRs & $f_{k}\left(\left\{\left[\mathbf{E}_{k}\right]_{n, n}\right\}_{n=1}^{N}\right)=\sum_{n=1}^{N} \frac{\left[\mathbf{E}_{k}\right]_{n, n}}{1-\left[\mathbf{E}_{k}\right]_{n, n}}=\sum_{n=1}^{N} \operatorname{SINR}_{k}^{-1}(n)$ \\
\hline The negative of the minimum of the SINRs & $\left.f_{k}\left(\left\{\left[\mathbf{E}_{k}\right]_{n, n}\right\}_{n=1}^{N}\right)=\max _{1 \leq n \leq N}\left[\mathbf{E}_{k}\right]_{n, n}=-\min _{1 \leq n \leq N} \operatorname{SINR}_{k}(n)\right)$ \\
\hline
\end{tabular}

\section{A. Linear Transceiver Design}

When a linear receiver is employed, the vector $\mathbf{r}$ is processed by a matrix $\mathbf{G}$ to obtain $\mathbf{y}=\mathbf{G H U} \mathbf{s}+\mathbf{G n}$. The MSE matrix $\mathbf{E}=\mathrm{E}\left\{(\mathbf{y}-\mathbf{s})(\mathbf{y}-\mathbf{s})^{H}\right\}$ turns out to be given by

$\mathbf{E}=\mathbf{I}_{K N}+\mathbf{G}\left(\mathbf{H U} \mathbf{U}^{H} \mathbf{H}^{H}+\mathbf{R}_{\mathbf{n}}\right) \mathbf{G}^{H}-\mathbf{G H U}-\mathbf{U}^{H} \mathbf{H}^{H} \mathbf{G}^{H}$

while the $k$ th MSE over the $n$th subcarrier is obtained as $\operatorname{MSE}_{k}(n)=[\mathbf{E}]_{(k-1) N+n,(k-1) N+n}$. For notational convenience, in all subsequent derivations we call

$$
\left[\mathbf{E}_{k}\right]_{n, n}=[\mathbf{E}]_{(k-1) N+n,(k-1) N+n}
$$

so that we may write $\operatorname{MSE}_{k}(n)=\left[\mathbf{E}_{k}\right]_{n, n}$.

Finding the optimal $\mathbf{G}$ reduces to look for a matrix that satisf es the QoS requirements for any given $\mathbf{U}$ and $\mathbf{F}$. Since $\left[\mathbf{E}_{k}\right]_{n, n}$ is a quadratic function of $\mathbf{G}$, the best we can do is to choose $\mathbf{G}_{\text {opt }}$ so as to minimize each MSE. Indeed, if such a matrix does not satisfy the QoS requirements no other one will [17]. As is well known, this is achieved by choosing $\mathbf{G}_{o p t}$ equal to the Wiener flter. In these circumstances, the MSE matrix in (9) takes the form

$$
\mathbf{E}=\mathbf{I}_{K N}-\mathbf{U}^{H} \mathbf{H}^{H}\left(\mathbf{H} \mathbf{U} \mathbf{U}^{H} \mathbf{H}^{H}+\mathbf{R}_{\mathbf{n}}\right)^{-1} \mathbf{H} \mathbf{U} .
$$

Now, we proceed with the design of the matrices $\mathbf{U}$ and $\mathbf{F}$ that solve

$$
\left(\mathcal{P}_{1}\right): \min _{\mathbf{U}, \mathbf{F}} P_{T} \quad \text { s.t. } \quad f_{k}\left(\left\{\left[\mathbf{E}_{k}\right]_{n, n}\right\}_{n=1}^{N}\right) \leq \gamma_{k} \quad \forall k
$$

with $\mathbf{E}$ given by (11). As mentioned before, closed-form solutions for $(\mathbf{U}, \mathbf{F})$ are now computed for $f_{k}$ being additively Schur-convex. A short list of such functions is given in Table I where we have used the fact that when the Wiener flter is used at the destination the signal-to-interference noise ratio (SINR) of the $k$ th stream over the $n$th subcarrier is given by $\operatorname{SINR}_{k}(n)=1 /\left[\mathbf{E}_{k}\right]_{n, n}-1$.

Proposition 1: If each $f_{k}$ is additively Schur-convex, the optimal matrices $\mathbf{U}_{\text {opt }}$ and $\mathbf{F}_{\text {opt }}$ in (12) are given by

$$
\mathbf{U}_{o p t}=\tilde{\mathbf{V}}_{H_{1}} \boldsymbol{\Lambda}_{U}^{1 / 2} \mathbf{S}^{H} \quad \text { and } \quad \mathbf{F}_{o p t}=\tilde{\mathbf{V}}_{H_{2}} \Lambda_{F}^{1 / 2} \tilde{\Omega}_{H_{1}}^{H}
$$

where $\tilde{\mathbf{V}}_{H_{1}}, \tilde{\mathbf{V}}_{H_{2}}$ and $\tilde{\boldsymbol{\Omega}}_{H_{1}}$ correspond to the $K N$ columns of $\mathbf{V}_{H_{1}}, \mathbf{V}_{H_{2}}$, and $\boldsymbol{\Omega}_{H_{1}}$ associated to the $K N$ largest singular values of the corresponding channel matrix while $\mathbf{S} \in \mathbb{C}^{K N \times K N}$ is a suitable unitary matrix such that

$$
\left[\mathbf{E}_{k}\right]_{n, n}=\epsilon_{k} \quad \forall n \in \mathcal{N}
$$

with $\epsilon_{k}$ obtained as

$$
\gamma_{k}=f_{k}(\underbrace{\epsilon_{k}, \epsilon_{k}, \ldots, \epsilon_{k}}_{N \text { times }}) .
$$

In addition, $\boldsymbol{\Lambda}_{U}=\operatorname{diag}\left\{\lambda_{U, 1}, \lambda_{U, 2}, \ldots, \lambda_{U, K N}\right\}$ and $\boldsymbol{\Lambda}_{F}=$ $\operatorname{diag}\left\{\lambda_{F, 1}, \lambda_{F, 2}, \ldots, \lambda_{F, K N}\right\}$ with elements in decreasing order.

Proof: See Appendix.

The above result represents one of the major contributions of this work and, to the best of our knowledge, cannot be found in any other existing work. As in [11], it follows that $\mathbf{U}_{\text {opt }}$ and $\mathbf{F}_{\text {opt }}$ match the singular vectors of the corresponding channel matrices. Then, the optimal structure of the overall communication system turns out to be diagonal up to a unitary matrix $\mathbf{S}$ that differently from [11] must be chosen so as to guarantee that the diagonal elements of $\mathbf{E}_{k}$ for $k=1,2, \ldots, K$ are all equal to $\epsilon_{k}$. The latter is always such that ${ }^{3} 0<\epsilon_{k}<1$ and it is computed through (15) on the basis of the given $\gamma_{k}$ and $f_{k}$. Assume for example that $f_{k}$ is the arithmetic mean of the MSEs, then $\epsilon_{k}$ results given by $\epsilon_{k}=\gamma_{k} / N$. On the other hand, $\epsilon_{k}=\gamma_{k}$ when $f_{k}$ takes the maximum of the MSEs over all subcarriers. Once all the quantities $\epsilon_{k}$ are computed, the unitary matrix $\mathbf{S}$ can be determined using the iterative procedure described in [20].

As shown in [11], the entries of $\boldsymbol{\Lambda}_{U}$ and $\boldsymbol{\Lambda}_{F}$ are obtained as the solutions of the following problem:

$$
\begin{array}{r}
\min _{\left\{\lambda_{U, i} \geq 0\right\},\left\{\lambda_{F, i} \geq 0\right\}} \sum_{i=1}^{K N}\left[\lambda_{U, i}+\lambda_{F, i}\left(\lambda_{U, i} \lambda_{H_{1}, i}+\rho_{1}\right)\right] \\
\text { s.t. } \quad \sum_{i=1}^{j} \lambda_{E, i} \leq \sum_{i=1}^{j} \eta_{i} \text { for } j=1,2, \ldots, K N
\end{array}
$$

where $\eta_{i}$ is def ned as $\eta_{i}=\epsilon_{\nu}$ with $\nu \in\{1,2, \ldots, K\}$ being the integer such that $(\nu-1) N<i \leq \nu N$, while $\lambda_{E, i}$ denotes the $i$ th eigenvalue of $\mathbf{E}$. Finding the solution of the above problem is hard since it is not in a convex form. To overcome this problem, one may resort to the algorithms developed in [11] in which the optimal solution of both problems is upper- and lower-bounded using the geometric programming approach and the dual decomposition technique, respectively. Unfortunately, the computational complexity of both algorithms is relatively high so as to make them unsuited for practical implementation. For this reason, in [15] the authors develop an alternative solution in which the nonconvex power allocation problems in (16) is approximated with a convex one that can be solved exactly through a multi-step

\footnotetext{
${ }^{3}$ Observe that $\epsilon_{k}$ must be larger than zero since a zero MSE can only be achieved when the noise is absent. Viceversa, it must be smaller than 1 otherwise we could satisfy the QoS constraint simply neglecting the transmission of the $k$ th stream.
} 
procedure of reduced complexity ${ }^{4}$.

In practical applications source and relay may be unable to meet all the QoS requirements due to their limited power resource or due to regulations specifying the maximum transmit power. This calls for some countermeasures. A possible way out to this problem (not investigated yet) is represented by the technique illustrated in [19] for single-hop MIMO systems in which the QoS constraints that produce the largest increase in terms of transmit power are frst identif ed and then relaxed using a perturbation analysis. An alternative approach is to make use of an admission control algorithm such as the one illustrated in [21] for multi-user single-antenna relay systems in which the power minimization problem is carried out jointly with the maximization of the number of users that can be QoSguaranteed.

\section{B. Non-linear Transceiver Design}

When a non-linear receiver with a decision-feedback equalizer is employed at the destination, the vector $\mathbf{z}$ at the input of the decision device (assuming correct previous decisions) can be written as $\mathbf{z}=(\mathbf{G H U}-\mathbf{B}) \mathbf{s}+\mathbf{G n}$ where $\mathbf{B} \in \mathbb{C}^{K N \times K N}$ is a strictly upper triangular matrix [9]. The MSE matrix takes the form

$$
\mathbf{E}=(\mathbf{G H U}-\mathbf{C})(\mathbf{G H U}-\mathbf{C})^{H}+\mathbf{G R}_{\mathbf{n}} \mathbf{G}^{H}
$$

where $\mathbf{C}=\mathbf{B}+\mathbf{I}_{K N}$ is a unit-diagonal upper triangular matrix. Using the same arguments adopted for the linear case, the optimal $\mathbf{G}$ is easily found to be such that each $\left[\mathbf{E}_{k}\right]_{n, n}$ is minimized. This yields [9]

$$
\mathbf{G}=\mathbf{C}\left(\mathbf{U}^{H} \mathbf{H}^{H} \mathbf{R}_{\mathbf{n}}^{-1} \mathbf{H} \mathbf{U}+\mathbf{I}_{K N}\right)^{-1} \mathbf{U}^{H} \mathbf{H}^{H} \mathbf{R}_{\mathbf{n}}^{-1} .
$$

We substitute (18) into (17) to obtain

$$
\mathbf{E}=\mathbf{C}\left(\mathbf{U}^{H} \mathbf{H}^{H} \mathbf{R}_{\mathbf{n}}^{-1} \mathbf{H} \mathbf{U}+\mathbf{I}_{K N}\right)^{-1} \mathbf{C}^{H}
$$

and look for the optimal $\mathbf{C}$. As for $\mathbf{G}$, the optimal $\mathbf{C}$ must be designed so as to minimize each $\left[\mathbf{E}_{k}\right]_{n, n}$. Following [9], this is achieved when $\mathbf{C}=\mathbf{D} \mathbf{L}^{H}$ where $\mathbf{L}$ is the lower triangular matrix obtained from the Cholesky decomposition of $\mathbf{U}^{H} \mathbf{H}^{H} \mathbf{R}_{\mathbf{n}}^{-1} \mathbf{H U}+\mathbf{I}_{K N}$ while the $K N \times K N$ diagonal matrix $\mathbf{D}$ is designed such that $[\mathbf{C}]_{i, i}=1$ for $i=1,2, \ldots, K N$. Once $\mathbf{C}$ has been computed, $\mathbf{B}$ is obtained as $\mathbf{B}=\mathbf{C}-\mathbf{I}_{K N}$. Using all the above results, it follows that [11]

$$
\left[\mathbf{E}_{k}\right]_{n, n}=1 /\left[\mathbf{L}_{k}\right]_{n, n}^{2}
$$

where $\left[\mathbf{L}_{k}\right]_{n, n}=[\mathbf{L}]_{(k-1) N+n,(k-1) N+n}$.

The design of $\mathbf{U}$ and $\mathbf{F}$ requires to solve (12) with $\left[\mathbf{E}_{k}\right]_{n, n}$ given by (20). Closed-form solutions for $\mathbf{U}$ and $\mathbf{F}$ are now computed for multiplicatively Schur-convex functions. Due to space limitations, we do not report a list of multiplicatively Schur-convex functions and limit to observe that every increasing additively Schur-convex function is multiplicatively Schur-convex as well [18]. Consequently, the additively Schurconvex functions reported in Table I can easily be accommodated in the following framework (see [17] for more details).

\footnotetext{
${ }^{4}$ It is worth observing that the suboptimal procedure developed in [15] must be seen as a means to approximate the solution of the arising power allocation problem rather than an alternative to compare with.
}

Proposition 2: If each $f_{k}$ is multiplicatively Schur-convex, then the optimal matrices $\mathbf{U}_{\text {opt }}$ and $\mathbf{F}_{\text {opt }}$ are given by

$$
\mathbf{U}_{o p t}=\tilde{\mathbf{V}}_{H_{1}} \boldsymbol{\Lambda}_{U}^{1 / 2} \mathbf{P}^{H} \quad \text { and } \quad \mathbf{F}_{o p t}=\tilde{\mathbf{V}}_{H_{2}} \boldsymbol{\Lambda}_{F}^{1 / 2} \tilde{\Omega}_{H_{1}}^{H}
$$

where $\mathbf{P} \in \mathbb{C}^{K N \times K N}$ is unitary and such that

$$
\left[\mathbf{L}_{k}\right]_{n, n}^{-1}=\sqrt{\epsilon_{k}} \text { for } n=1,2, \ldots, N
$$

with $\epsilon_{k}$ for $k=1,2, \ldots, K$ still given by (15). In addition, the matrices $\boldsymbol{\Lambda}_{U}$ and $\boldsymbol{\Lambda}_{F}$ are diagonal with elements in decreasing order.

Proof: See Appendix.

As for the linear case, it turns out that channel-diagonalizing structure is optimal provided that the symbols are properly rotated by the unitary matrix $\mathbf{P}$. The latter must be now chosen such that (22) is satisf ed. This can be achieved resorting to the algorithm illustrated in [17].

The entries of $\boldsymbol{\Lambda}_{U}$ and $\boldsymbol{\Lambda}_{F}$ are now solutions of the following power allocation problem

$$
\begin{aligned}
\min _{\left\{\lambda_{U, i} \geq 0\right\},\left\{\lambda_{F, i} \geq 0\right\}} \sum_{i=1}^{K N}\left[\lambda_{U, i}+\lambda_{F, i}\left(\lambda_{U, i} \lambda_{H_{1}, i}+\rho_{1}\right)\right] \\
\text { s.t. } \prod_{i=1}^{j} \lambda_{E, i} \leq \prod_{i=1}^{j} \eta_{i} \text { for } j=1,2, \ldots, K N
\end{aligned}
$$

where $\eta_{i}$ is def ned as in Proposition 1. A close inspection of (16) and (23) reveal that the two power allocation problems differ for the inequality constraints. As before, the above problem is not in a convex form and its solution can be closely approximated resorting to the power allocation algorithms discussed in [11] and [15].

\section{NUMERICAL RESUlts}

Numerical results are now given to assess the performance of the proposed solutions. The OFDM terminals employ discrete Fourier transform units of size $N_{T}=512$ with a cyclic pref $x$ composed of 32 samples and transmit over a bandwidth of $20 \mathrm{MHz}$. Two different stream are supported over $N=32$ subcarriers. The number of antennas is $N_{S}=N_{R}=3$. The transmitted symbols belong to a 4-QAM constellation. The channel taps are generated as specif ed in the ITU IMT2000 Vehicular-A channel model. The transmit and receive antennas are assumed to be adequately separated so as to make the channel realizations statistically independent in the spatial domain. Comparisons are made with SA (suboptimal approach) in which the unitary matrices $\mathbf{S}$ and $\mathbf{P}$ in (13) and (21) are set equal to the identity matrix (see [11] - [14]).

Fig. 1 illustrates the total power consumption as a function of the QoS constraints when the noise variance over both links is equal and given by 1 or 0.01 . For illustrative reasons, the same QoS constraint is imposed for each class of service. This amounts to saying that $\gamma_{k}=\gamma$ for $k=1,2$. Assume for example that $f_{k}$ is the arithmetic mean of the MSEs then $\epsilon_{k}=\gamma / N$ for $k=1,2$. On the other hand, if $f_{k}$ is the maximum MSE then $\epsilon_{k}=\gamma$ for $k=1,2$. The curves labelled with RC-L and RC-NL refer respectively to a system in which a linear or a nonlinear receiver is employed in conjunction with the reduced-complexity power allocation algorithm proposed 


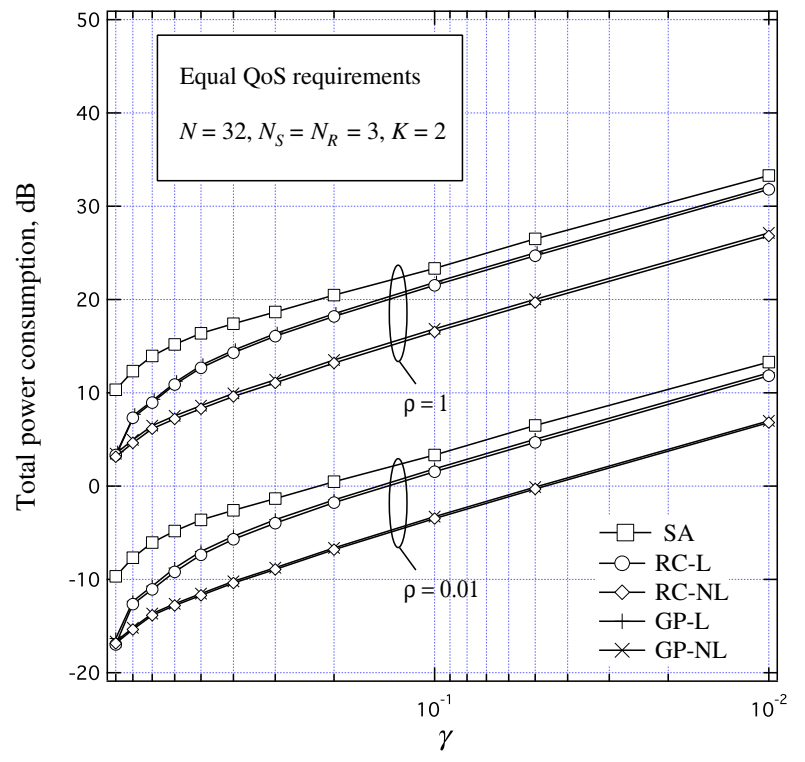

Fig. 1. Total power consumption when equal QoS constraints are given with $N=32, N_{S}=N_{R}=3, K=2$ and $\rho=1$ or 0.01 .

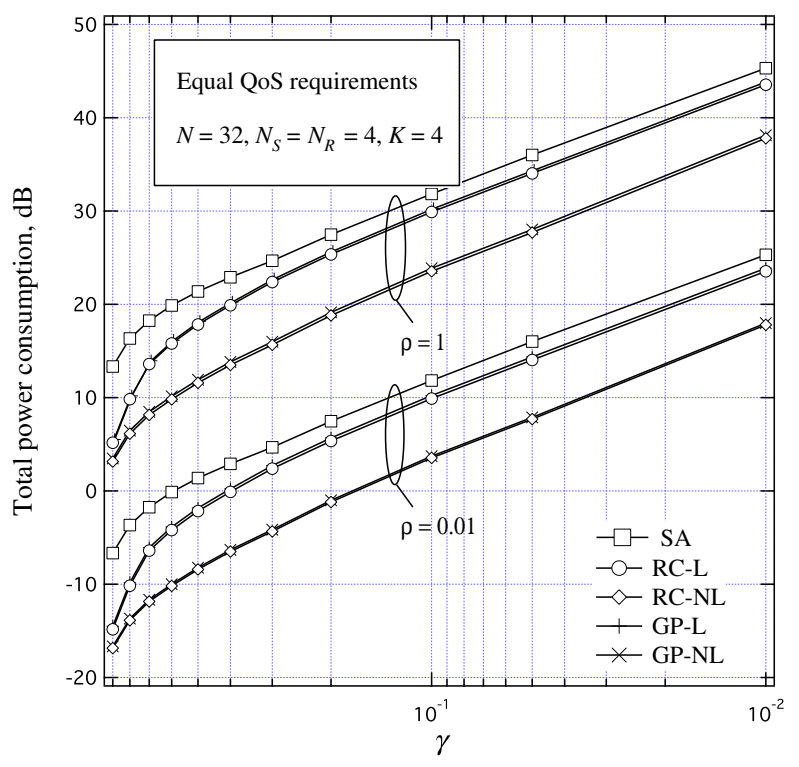

Fig. 2. Total power consumption when equal QoS constraints are given with $N=32, N_{S}=N_{R}=4, K=4$ and $\rho=1$ or 0.01 .

in [15]. On the other hand, GP-L and GP-NL refer to a system in which the successive geometric programming (GP) approach of [11] is employed in conjunction with a linear or a nonlinear receiver, respectively. The results of Fig. 1 indicate that the optimization leads to a remarkable gain with respect to SA and that the non-linear architecture provides the best performance for all the investigated values of $\gamma$. As seen, the total power consumption required by [15] is substantially the same as that obtained with the solution discussed in [11]. Similar conclusions can be drawn from the results of Fig. 2 in which $N_{S}=N_{R}=4$ and $K=4$.

The results of Fig. 3 are obtained in the same operating conditions of Fig. 2 except that now $\gamma_{1}=\gamma, \gamma_{2}=\gamma / 8$

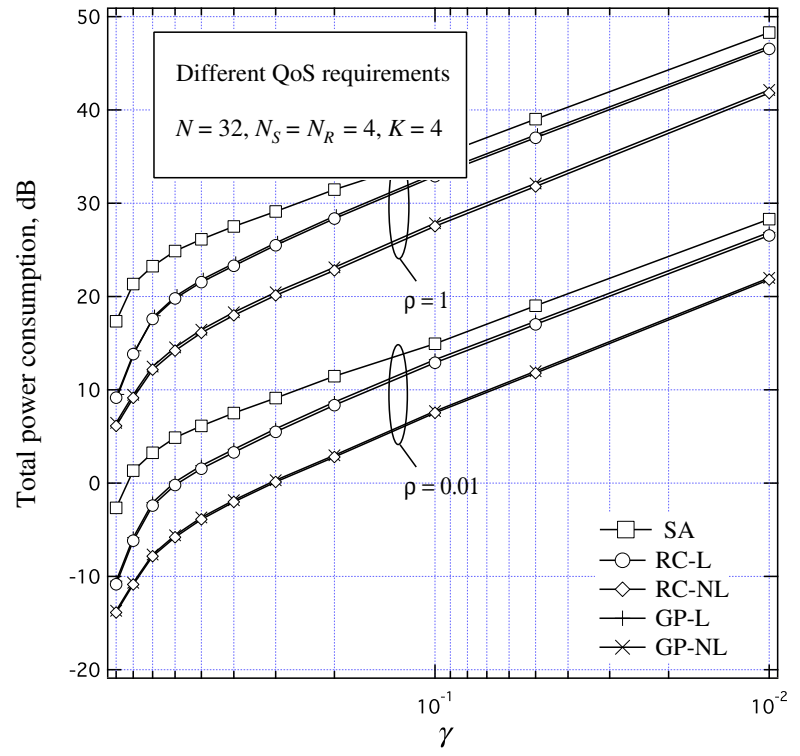

Fig. 3. Total power consumption when different QoS constraints are given with $N=32, N_{S}=N_{R}=4, K=4$ and $\rho=1$ or 0.01 .

and $\gamma_{3}=\gamma_{4}=\gamma / 6$. Compared to the results of Fig. 2, the total power consumption increases due to the more stringent requirements over some established links.

\section{CONCLUSIONS}

We have discussed the optimization of linear and nonlinear architectures for MIMO-OFDM relay networks to minimize the total power consumption while satisfying QoS requirements given as additively/multiplicatively Schur-convex functions of the MSEs of each stream over all subcarriers. Interestingly, it is found that for both classes of functions the diagonalizing structure is optimal provided that the transmitted data symbols are properly rotated before channel diagonalization.

\section{APPENDIX}

The proof of Proposition 1 relies on showing that if each $f_{k}$ is additively Schur-convex then the original problem $\left(\mathcal{P}_{1}\right)$ in (12) is equivalent to the following one $\left(\mathcal{P}_{2}\right)$ :

$$
\left(\mathcal{P}_{2}\right): \min _{\mathbf{U}, \mathbf{F}} P_{T} \quad \text { s.t. } \quad\left[\mathbf{E}_{k}\right]_{1,1}=\cdots=\left[\mathbf{E}_{k}\right]_{N, N} \leq \epsilon_{k} \forall k
$$

where $P_{T}$ is given by (8) and $\epsilon_{k}$ is such that

$$
f_{k}\left(\mathbf{1}_{\epsilon_{k}}\right)=\gamma_{k}
$$

with $\mathbf{1}_{\epsilon_{k}}$ being the $N$-dimensional vector def ned as $\mathbf{1}_{\epsilon_{k}}=$ $\left[\epsilon_{k}, \epsilon_{k}, \ldots, \epsilon_{k}\right]^{T}$. The above problem is formally equivalent to the one discussed in [11] meaning that the matrices $\mathbf{U}$ and $\mathbf{F}$ solving $\left(\mathcal{P}_{2}\right)$ have the same form of those computed in [11] and are given by (13) in the text.

For notational convenience, we denote by $P_{T}(\mathbf{U}, \mathbf{F})$ the transmit power required by the matrices $(\mathbf{U}, \mathbf{F})$ and call $\left[\mathbf{E}_{k}(\mathbf{U}, \mathbf{F})\right]_{n, n}$ the corresponding MSE of the $k$ th symbol over the $n$th subcarrier. 
To establish the equivalence of $\left(\mathcal{P}_{1}\right)$ and $\left(\mathcal{P}_{2}\right)$, it is enough to show that for any pair $\left(\mathbf{U}_{1}, \mathbf{F}_{1}\right)$ in the feasible set of $\left(\mathcal{P}_{1}\right)$ it is always possible to $\mathrm{fnd}$ a corresponding pair $\left(\mathbf{U}_{2}, \mathbf{F}_{2}\right)$ in the feasible set of $\left(\mathcal{P}_{2}\right)$ for which the same transmit power is required, i.e., $P_{T}\left(\mathbf{U}_{1}, \mathbf{F}_{1}\right)=P_{T}\left(\mathbf{U}_{2}, \mathbf{F}_{2}\right)$ and vice-versa. We start assuming that $\left(\mathbf{U}_{1}, \mathbf{F}_{1}\right)$ is in the feasible set of $\left(\mathcal{P}_{1}\right)$, i.e.,

$$
f_{k}\left(\left\{\left[\mathbf{E}_{k}\left(\mathbf{U}_{1}, \mathbf{F}_{1}\right)\right]_{n, n}\right\}_{n=1}^{N}\right) \leq \gamma_{k} .
$$

Using the results illustrated [20], it can be shown that there always exists a unitary matrix $\mathbf{S}$ such that the MSEs become all equal to their arithmetic mean, i.e.,

$$
\left[\mathbf{E}_{k}\left(\mathbf{U}_{1} \mathbf{S}, \mathbf{F}_{1}\right)\right]_{n, n}=\frac{1}{N} \sum_{j=1}^{N}\left[\mathbf{E}_{k}\left(\mathbf{U}_{1}, \mathbf{F}_{1}\right)\right]_{j, j}=\theta_{k} .
$$

To proceed further, denote by $\mathbf{e}_{k}\left(\mathbf{U}_{1}, \mathbf{F}_{1}\right)$ the vector collecting the MSEs of the $k$ th stream, i.e., $\mathbf{e}_{k}\left(\mathbf{U}_{1}, \mathbf{F}_{1}\right)=$ $\left[\left[\mathbf{E}_{k}\left(\mathbf{U}_{1}, \mathbf{F}_{1}\right)\right]_{1,1}, \quad\left[\mathbf{E}_{k}\left(\mathbf{U}_{1}, \mathbf{F}_{1}\right)\right]_{2,2}, \ldots,\left[\mathbf{E}_{k}\left(\mathbf{U}_{1}, \mathbf{F}_{1}\right)\right]_{N, N}\right]^{T}$. From [22], it is seen that that $\mathbf{1}_{\theta_{k}} \prec_{+} \mathbf{e}_{k}\left(\mathbf{U}_{1}, \mathbf{F}_{1}\right)$ where $\mathbf{1}_{\theta_{k}}$ is the $N$-dimensional vector def ned as $\mathbf{1}_{\theta_{k}}=$ $\left[\theta_{k}, \theta_{k}, \ldots, \theta_{k}\right]^{T}$. If $f_{k}$ is additively Schur-convex, then $f_{k}\left(\mathbf{1}_{\theta_{k}}\right) \leq f_{k}\left(\mathbf{e}_{k}\left(\mathbf{U}_{1}, \mathbf{F}_{1}\right)\right)$ from which using (25) it follows that $f_{k}\left(\mathbf{1}_{\theta_{k}}\right) \leq \gamma_{k}$ or, equivalently, $f_{k}\left(\mathbf{1}_{\theta_{k}}\right) \leq f_{k}\left(\mathbf{1}_{\epsilon_{k}}\right)$ where we have used the def nition in (24). Since $f_{k}$ is a nondecreasing function of its arguments, from $f_{k}\left(\mathbf{1}_{\theta_{k}}\right) \leq f_{k}\left(\mathbf{1}_{\epsilon_{k}}\right)$ it follows that $\left[\mathbf{E}_{k}\left(\mathbf{U}_{1} \mathbf{S}, \mathbf{F}_{1}\right)\right]_{n, n}=\theta_{k} \leq \epsilon_{k}$ which amounts to saying that $\left(\mathbf{U}_{1} \mathbf{S}, \mathbf{F}_{1}\right)$ is in the feasible set of $\left(\mathcal{P}_{2}\right)$. In addition, from (8) it easily follows that $P_{T}\left(\mathbf{U}_{1}, \mathbf{F}_{1}\right)=P_{T}\left(\mathbf{U}_{1} \mathbf{S}, \mathbf{F}_{1}\right)$. Then, we may conclude that for any feasible $\left(\mathbf{U}_{1}, \mathbf{F}_{1}\right)$ in $\left(\mathcal{P}_{1}\right)$ there always exists a pair $\left(\mathbf{U}_{2}, \mathbf{F}_{2}\right)$ of the form $\left(\mathbf{U}_{2}, \mathbf{F}_{2}\right)=$ $\left(\mathbf{U}_{1} \mathbf{S}, \mathbf{F}_{1}\right)$, which is in the feasible set of $\left(\mathcal{P}_{2}\right)$ and requires the same amount of transmit power.

We now prove the reverse part. Let $\left(\mathbf{U}_{2}, \mathbf{F}_{2}\right)$ be in the feasible set of $\left(\mathcal{P}_{2}\right)$, i.e.,

$$
\left[\mathbf{E}_{k}\left(\mathbf{U}_{2}, \mathbf{F}_{2}\right)\right]_{1,1}=\cdots=\left[\mathbf{E}_{k}\left(\mathbf{U}_{2}, \mathbf{F}_{2}\right)\right]_{N, N} \leq \epsilon_{n}
$$

with required transmit power $P_{T}\left(\mathbf{U}_{2}, \mathbf{F}_{2}\right)$. Letting $\left[\mathbf{E}_{k}\left(\mathbf{U}_{2}, \mathbf{F}_{2}\right)\right]_{n, n}=\theta_{k} \forall n$ and exploiting the fact that $f_{k}$ is a non-decreasing function of its arguments, using (24) and (27) we may write

$$
f_{k}\left(\left\{\left[\mathbf{E}_{k}\left(\mathbf{U}_{2}, \mathbf{F}_{2}\right)\right]_{n, n}\right\}_{n=1}^{N}\right)=f_{k}\left(\mathbf{1}_{\theta_{k}}\right) \leq f_{k}\left(\mathbf{1}_{\epsilon_{k}}\right)=\gamma_{k}
$$

from which it follows that $\left(\mathbf{U}_{2}, \mathbf{F}_{2}\right)$ is in the feasible set of $\left(\mathcal{P}_{1}\right)$. Therefore, setting $\left(\mathbf{U}_{1}, \mathbf{F}_{1}\right)=\left(\mathbf{U}_{2}, \mathbf{F}_{2}\right)$ yields the desired result. This completes the proof of Proposition 1.

The proof of Proposition 2 is much similar to that of Proposition 1. For this reason, in the sequel we report only the major differences. The frst part relies on the observation that it is always possible to $\mathrm{f}$ nd a unitary matrix $\mathbf{P}$ such that the MSEs given by (20) become all equal to their geometric mean [17], i.e.,

$$
\left[\mathbf{E}_{k}\left(\mathbf{U}_{1} \mathbf{P}, \mathbf{F}_{1}\right)\right]_{n, n}=\left(\prod_{j=1}^{N}\left[\mathbf{E}_{k}\left(\mathbf{U}_{1}, \mathbf{F}_{1}\right)\right]_{j, j}\right)^{\frac{1}{N}}=\theta_{n} .
$$

In addition, if $f_{k}$ is multiplicatively Schur-convex then $f_{k}\left(\mathbf{1}_{\theta_{k}}\right) \leq f_{k}\left(\mathbf{e}_{k}\left(\mathbf{U}_{1}, \mathbf{F}_{1}\right)\right)$ from which using the same arguments of before it easily follows that $\left(\mathbf{U}_{1} \mathbf{P}, \mathbf{F}_{1}\right)$ is in the feasible set of $\left(\mathcal{P}_{2}\right)$ and requires the same amount of power. The reverse part is straightforward.

\section{REFERENCES}

[1] A. Nosratinia, T. E. Hunter, A. Hedayat, "Cooperative communication in wireless networks," IEEE Commun. Mag., vol. 42, no. 10, pp. $74-$ 80 , Oct. 2004

[2] G. Kramer, M. Gastpar, and P. Gupta, "Cooperative strategies and capacity theorems for relay networks," IEEE Trans. Inf. Theory, vol. 51, no. 9, pp. 3037 - 3063, Sept. 2005.

[3] 3GPP Tech. Spec. Group, "Radio Access Network; Evolved Universal Terrestrial Radio Access (E-UTRA); Further advancements for EUTRA: Physical layer aspects," Tech. Rep. 3GPP TR36.814 v1.2.1, June 2009.

[4] IEEE 802.16 Broadband Wireless Access Working Group, "IEEE 802.16j Amendment 1: Multihop Relay Specif cation,” Tech. Rep. IEEE 802.16j-2009, June 2009

[5] X. Tang and Y. Hua, "Optimal design of non-regenerative MIMO wireless relays," IEEE Trans. Wireless Commun., vol. 6, no. 4, pp. 1398 - 1407, April 2007.

[6] O. Munoz-Medina, J. Vidal, and A. Agustin, "Linear transceiver design in nonregenerative relays with channel state information," IEEE Trans. Signal Process., vol. 55, no. 6, pp. 2593 - 2604, June 2007.

[7] W. Guan, H. Luo, and W. Chen, "Linear relaying scheme for MIMO relay system with QoS requirements," IEEE Signal Process. Lett., vol. 15 , pp. $697-700,2008$.

[8] Y. Rong and Y. Hua, "Optimality of diagonalization of multi-hop MIMO relays," IEEE Trans. Wireless Commun., vol. 8, no. 12, pp. 6068 - 6077, 2009.

[9] Y. Rong, "Optimal linear non-regenerative multi-hop MIMO relays with MMSE-DFE receiver at the destination," IEEE Trans. Wireless Commun., vol. 9, no. 7, pp. 2268 - 2279, July 2010.

[10] Y. Hua, "An overview of beamforming and power allocation for MIMO relays," in Proc. IEEE MILCOM, San Jose, CA, Oct. 31 Nov. 03, 2010.

[11] Y. Rong, "Multihop non-regenerative MIMO relays: QoS considerations," IEEE Trans. Signal Process., vol. 59, no. 1, pp. 290 - 303, 2011.

[12] W. Zhang, U. Mitra, and M. Chiang, "Optimization of amplify-andforward multicarrier two-hop transmission," IEEE Trans. Commun., vol. 59, no. 5, pp. 14341445, May, 2011.

[13] K. Doppler and A. Hottinen, "Multi-antenna relay nodes in OFDM systems," in Proc. 3rd Int. Symp. Wireless Commun. Systems (ISWCS06), pp. 258 - 261, Valencia, Spain, 2006.

[14] Y. Rong, "Optimal multicarrier multi-hop non-regenerative MIMO relays with QoS constraints," in Proc. of the 12th Australian Commun. Theory Workshop, Melbourne, Australia, Jan. 312011 - Feb. 32011.

[15] L. Sanguinetti and A. A. D'Amico, "Power allocation in two-hop amplify-and-forward MIMO relay systems with QoS requirements," IEEE Trans. Signal Process., vol. 60, no. 5, pp. 2494 - 2507, May 2012.

[16] L. Sanguinetti, A. A. D'Amico and Y. Rong, "A tutorial on the optimization of amplify-and-forward MIMO relay systems," IEEE $J$. Select. Areas Commun., vol. 30, no. 8, pp. 1331 - 1346, Sept. 2012.

[17] D. P. Palomar and Y. Jiang, MIMO Transceiver Design via Majorization Theory. Now Publishers, vol. 3, no. 4-5, pp. 331-551: Foundations and Trends in Commun. and Inf. Theory, 2006.

[18] A. A. D'Amico, "Tomlinson-Harashima Precoding in MIMO Systems: A Unif ed Approach to Transceiver Optimization Based on Multiplicative Schur-Convexity," IEEE Trans. Signal Process., vol. 56, no. 8, pp. 3662 - 3677, Sept. 2008.

[19] D. P. Palomar, M. Lagunas, and J. Cioff, "Optimum linear joint transmitreceive processing for MIMO channels with QoS constraints," IEEE Trans. Signal Process., vol. 52, no. 5, pp. 1179 - 1197, May 2004.

[20] P. Viswanath and V. Anantharam, "Optimal sequences and sum capacity of synchronous CDMA systems," IEEE Trans. Inf. Theory, vol. 45, no. 6 , pp. 1984 - 1991, Sep. 1999.

[21] K. Phan, T. Le-Ngoc, S. Vorobyov, and C. Tellambura, "Power allocation in wireless multi-user relay networks," IEEE Trans. Wireless Commun., vol. 8, no. 5, pp. 2535 - 2545, May 2009.

[22] A. W. Marshall and I. Olkin, Inequalities: Theory of majorization, Ed. Academic press, 1979. 